1.02

\title{
Effect of Astroglial Necrosis on Blood-Brain Barrier Maintenance and Development in vivo
}

\author{
J.M. Krum \\ Department of Anatomy, The George Washington University Medical Center, \\ Washington, D.C. 20037, USA
}

In vitro, brain-derived endothelium loses several blood-brain barrier (BBB) characteristics such as $\gamma$-glutamyl transpeptidase, tight junctions and the polarized transport of amino acids. However, these features are regained during co-culture with astroglial cells or their conditioned medium. To determine if similar BBB deficits would occur in situ when microvessels were denuded of their astrocytic complement, chronic and acute perivascular astroglial destruction was effected by subcutaneous injection of the gliotoxin 6aminonicotinamide (6-AN) into neonatal and adult Wistar rats. Animals bearing neocortical transplants were also exposed to the toxin to test whether glial and/or barrier defects would occur within the grafts. 6-AN is a nicotinamide antagonist which causes a blockade of the pentose-phosphate pathway. Both astrocytes and oligodendrocytes are particularly susceptible to this toxin at the dosages used in this study (10 $\mathrm{mg} / \mathrm{kg}$ body weight), and they subsequently undergo cytotoxic edema followed by degeneration. The microvascular endothelium sustains no morphological damage after 6-AN administration.

Neonatal animals received 4 intraperitoneal injections of 6-AN during the first postnatal week and were sacrificed at ages ranging from 6 - 12 days. Age-matched controls received injections of saline. 6-AN caused necrosis of glial cells throughout the entire CNS in neonates resulting in the appearance of spongy degeneration in both white and gray matter. Perivascular astroglial processes were immensely swollen, with degenerating organelles and plasma membranes. The perivascular basement membrane appeared to be intact and stained normally with anti-laminin. Immunostaining for the glucose transporter protein and histochemical staining for $\gamma$-glutamyl transpeptidase revealed an expression of both $\mathrm{BBB}$ markers throughout the CNS of the experimental animals which was comparable to that of the age-matched controls. The $\mathrm{BBB}$ to endogenous protein remained intact as shown by immunostaining with rat serum albumin (RSA). Similarly, intravascularly injected horseradish peroxidase (HRP) did not leak from the microvasculature of experimental animals except in normally permeable areas and ultrastructural examination of these animals revealed a normal endocytotic uptake of HRP. Thus, despite widespread glial destruction throughout the neonatal CNS, the BBB characteristics of the developing vasculature were unaffected.

Adult rats received a single intraperitoneal injection of 6-AN and were sacrificed after 1 - 5 days. Although mature rats exhibited glial necrosis only in specific regions of spinal cord, medulla, cerebellum and thalamus, the microvascular ultrastructure of the affected regions was similar to that of 6-AN-exposed neonates. Petechial hemorrhages associated with veins were observed within the lesioned areas. Perivascular laminin immunoreactivity appeared to be normal. Endothelial immunoreactivity for the glucose transporter and the endothelial barrier antigen, an as yet uncharacterized $\mathrm{BBB}$ marker, also remained at normal levels. However, serum albumin extravasation was apparent in the affected CNS regions, and small areas of HRP leakage were also present, indicating that a breach of the BBB had occurred. Ultrastructural examination of the HRP leaks indicated that there was no increase in vesicular transport nor was there a conduit through the tight junctional complexes, thus allowing the conclusion that the observed protein leakage was the result of the venous hemorrhages observed within the 
affected CNS regions.

E19 fetal neocortex was transplanted to either the fourth ventricle or the parietal cortex of adult hosts. The animals received 6-AN injections at one week or one month postoperative and were sacrificed two days later. One week old transplants in either location were well vascularized and contained few degenerating glial cells when compared to the neocortex of 7-8 day old rat pups given a similar dosage of the gliotoxin. Glial cells within one month old transplants appeared completely intact and no $\mathrm{BBB}$ abnormalities were detected, although some reactive astroglia at the intraparenchymal interface region were damaged by 6-AN.
These results suggest that the induction and maintenance of some BBB properties in situ may not be completely dependent on astroglial contact as they are in vitro. However, because the microvascular basement membrane remains intact, the possibility that factors secreted by astrocytes may still reside there to maintain endothelial barrier properties cannot be ruled out.

Glial cells within neocortical transplants do not appear to be as susceptible to the effects of 6-AN as comparably aged neocortex in situ. This phenomenon may be the result of a change in glial cell metabolism within transplanted neocortex which may have significance in terms of their interactions with adjacent neurons. 

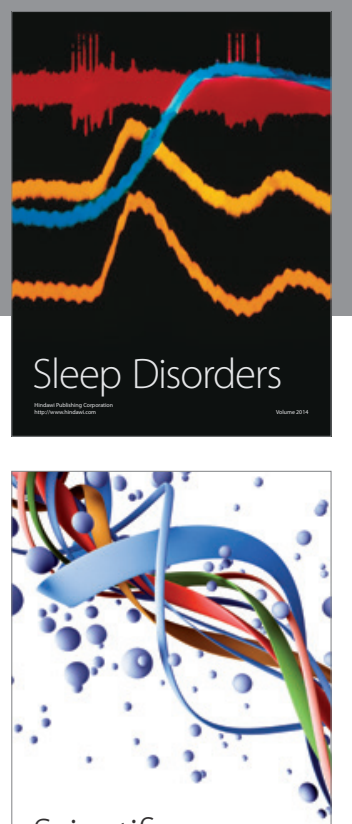

Scientifica
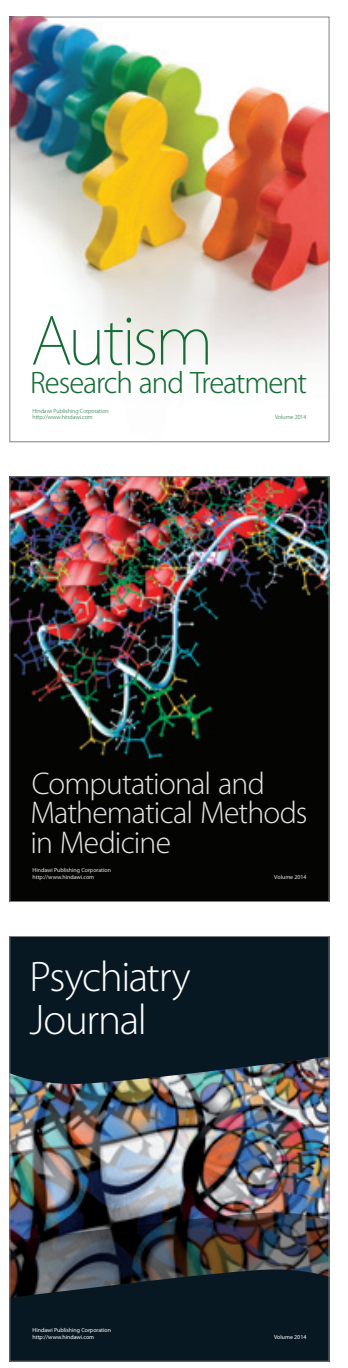
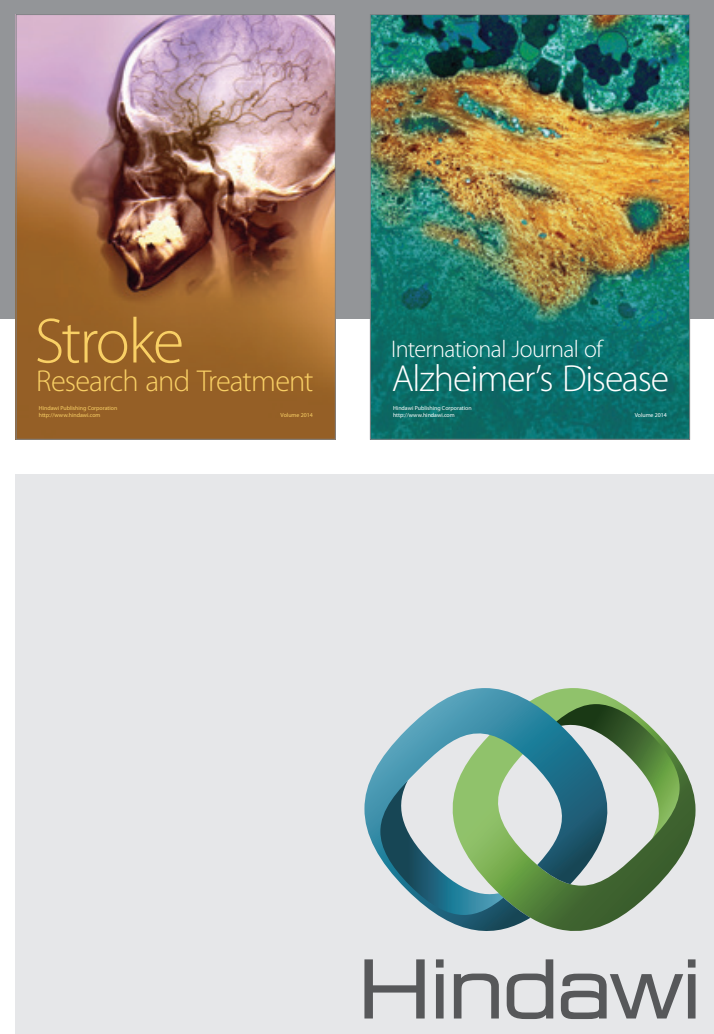

Submit your manuscripts at

http://www.hindawi.com
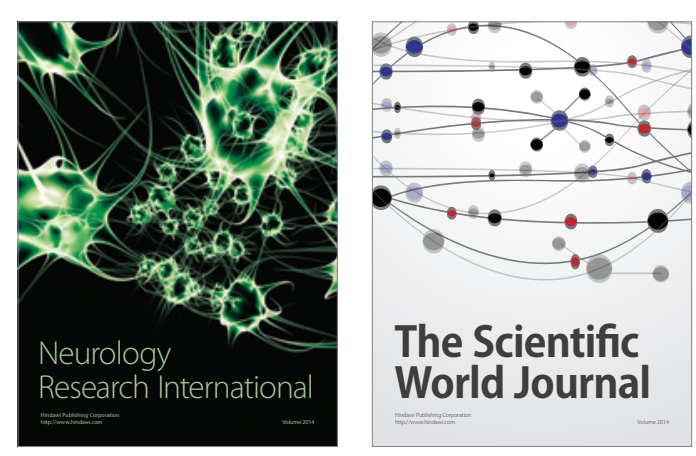

The Scientific World Journal

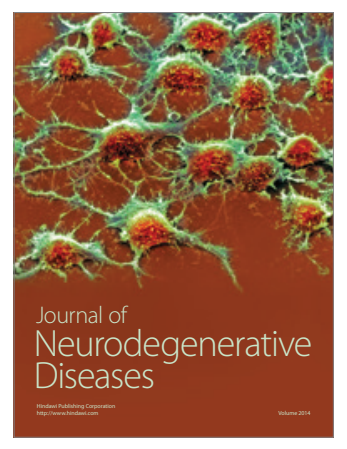

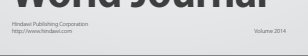

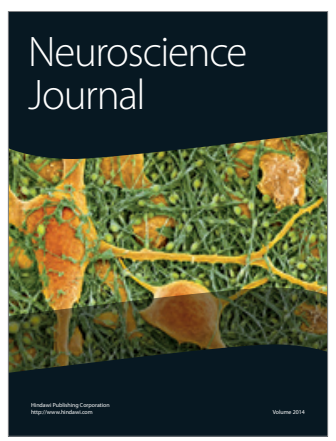

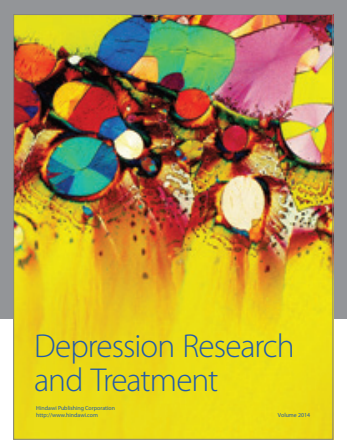
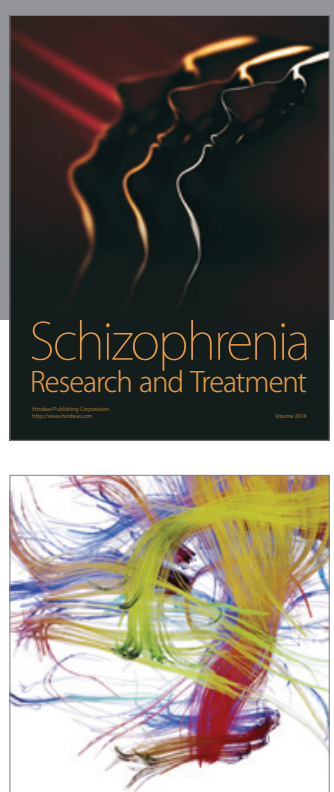

Brain Science

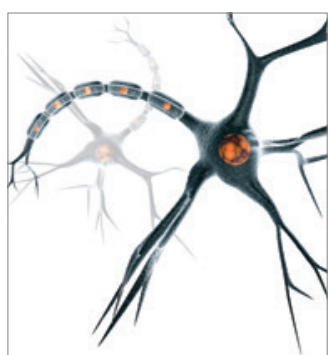

Neural Plasticity
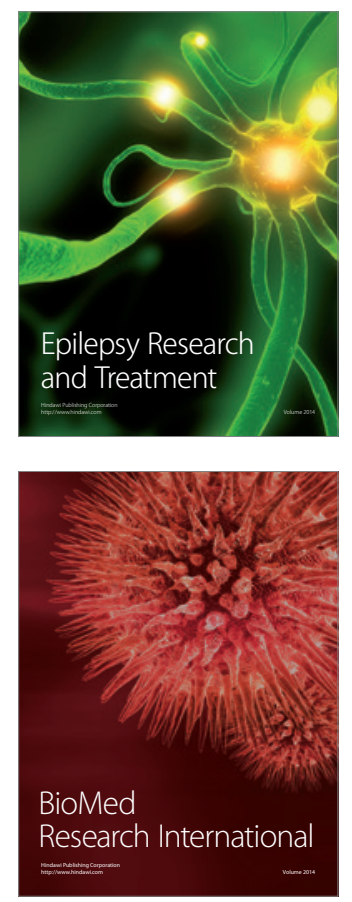

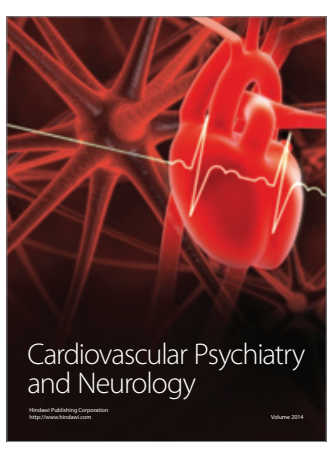

Parkinson's

Disease
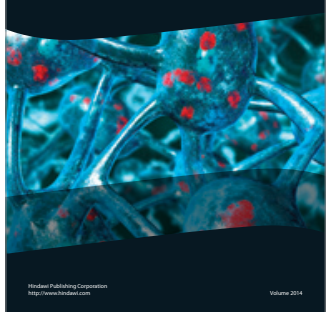